\title{
Extracellular Cation Binding Pocket Is Essential For Ion Conduction Of OsHKT2;2
}

\author{
Running title: Cation binding pocket of OsHKT2;2
}

\author{
Janin Riedelsberger ${ }^{1, *}$, Ariela Vergara-Jaque ${ }^{1,3}$, Miguel Piñeros ${ }^{2}$, Ingo Dreyer ${ }^{1}$, Wendy \\ González ${ }^{1,3}$ \\ ${ }^{1}$ Centro de Bioinformática y Simulación Molecular, Facultad de Ingeniería, Universidad de \\ Talca, Talca, Chile \\ ${ }^{2}$ Robert W. Holley Center for Agriculture and Health, USDA-ARS, Cornell University, Ithaca, \\ NY, United States \\ ${ }^{3}$ Millennium Nucleus of Ion Channels-Associated Diseases (MiNICAD), Chile \\ * Correspondence: \\ Janin Riedelsberger \\ jriedelsberger@utalca.cl
}

Keywords: ion channel, HKT, sodium transport, potassium transport, ion binding pocket, plant, structure-function

Words: 4,972

Figures: $8+3$ supplemental

\begin{abstract}
HKT channels mediate sodium uniport or sodium and potassium symport in plants. Monocotyledons express a higher number of HKT proteins than dicotyledons, and it is only within this clade of HKT channels that cation symport mechanisms are found. The prevailing ion composition in the extracellular medium affects the transport abilities of various HKT channels by changing their selectivity or ion transport rates. How this mutual effect is achieved at the molecular level is still unknown. Here, we built a homology model of the monocotyledonous OsHKT2;2, which shows sodium and potassium symport activity, and performed molecular dynamics simulations in the presence of sodium and potassium ions. By analyzing ion-protein interactions, we identified a cation binding pocket on the extracellular protein surface, which is formed by residues P71, D75, D501 and K504. Proline and the two aspartate residues coordinate cations, while K504 forms salt bridges with D75 and D501 and may be involved in the forwarding of cations towards the pore entrance. Functional validation via electrophysiological experiments confirmed the biological relevance of the predicted ion binding pocket and identified K504 as a central key residue. Mutation of the cation coordinating residues affected the functionality of HKT only slightly. Additional in silico mutants and simulations of K504 supported experimental results.
\end{abstract}

\section{Introduction}


A proper sodium $\left(\mathrm{Na}^{+}\right)$/potassium $\left(\mathrm{K}^{+}\right)$homeostasis is a crucial requirement for high yielding plant growth. Although $\mathrm{Na}^{+}$ions can promote plant growth at low concentrations, they turn hazardous at increasing levels (Leigh and Wyn Jones, 1984; Maathuis, 2009; Marschner, 2012). Due to similar physicochemical properties, $\mathrm{Na}^{+}$ions can mimic functions of $\mathrm{K}^{+}$ions, bridging periods of $\mathrm{K}^{+}$shortage (Benito et al., 2014; Horie et al., 2007). However, at high concentrations, $\mathrm{Na}^{+}$ions compete with $\mathrm{K}^{+}$and cause $\mathrm{K}^{+}$deficiency symptoms in plants, since $\mathrm{Na}^{+}$imitates $\mathrm{K}^{+}$' $\mathrm{s}$ functions only incompletely (Hasegawa et al., 2000; Pardo and Quintero, 2002).

Plants have developed sophisticated mechanisms to cope with salt stress. These systems aim to avoid high cytosolic $\mathrm{Na}^{+}$concentrations in cells of plant shoots by compartmentalization and retrieval of $\mathrm{Na}^{+}$from the xylem sap (Adams and Shin, 2014; Flowers and Läuchli, 1983). HKT channels form one comprehensive family that is involved in $\mathrm{Na}^{+}$usage and detoxification (Horie et al., 2009; Maser et al., 2002; Munns et al., 2012; Møller et al., 2009). In monocots, like rice, two types of HKT channels exist: (i) class I-type channels, which are mainly $\mathrm{Na}^{+}$selective, and (ii) channels of class II acting as $\mathrm{Na}^{+} / \mathrm{K}^{+}$co-transporters (Almeida et al., 2013; Garciadeblás et al., 2003). Among HKTs, there is a wide variety of transport kinetics, cation selectivity and rectification properties even within the two classes (Gassmann et al., 1996; Haro et al., 2005; Horie et al., 2001; Jabnoune et al., 2009; Rubio et al., 1995; Yao et al., 2010).

Several studies have demonstrated the interactive effect of different ion species for various HKT channels. In particular, the mutual effects of $\mathrm{Na}^{+}$and $\mathrm{K}^{+}$have been studied (Horie et al., 2001; Oomen et al., 2012; Yao et al., 2010). However, the mechanism by which ion species affect each other and modulate the ion transport process is so far not understood. Initially, it was discussed that HKT channels form multi-ion pores (Gassmann et al., 1996; Yao et al., 2010). In this scenario, multiple ion binding sites exist in the pore allowing a coupled ion transport without additional conformational changes ( $\mathrm{Su}$ et al., 1996). However, recently Böhm and colleagues experimentally demonstrated that in the Venus flytrap HKT1 a maximum of one ion at a time occupies the selectivity filter (Böhm et al., 2015). Therefore, the mechanism behind mutual ion species effects remains controversial.

In this study, we provide new functional-structural perspectives for the understanding of ion species-specific effects on ion conduction in HKT channels. We built a homology model of the Oryza sativa HKT channel OsHKT2;2 embedded in a lipid membrane, performed molecular dynamics simulations in the presence of $\mathrm{Na}^{+}$and $\mathrm{K}^{+}$ions and evaluated protein-ion interactions. Through systematic analysis, we identified a potential cation binding pocket at the extracellular surface of the protein - a region that ions have to pass before entering the pore. The cation binding pocket was characterized by computational studies and its functional importance for OsHKT2;2 was experimentally validated using electrophysiological methods. Additionally, analyses of in silico mutants further underpinned the experimental insights.

\section{Materials and Methods}

\subsection{Homology modelling and molecular dynamics simulation}

The structural model of $O s H K T 2 ; 2$ was generated using the I-Tasser server (Roy et al., 2010). Full-length structural models were built from multiple threading alignments. For threading the bacterial channels KtrB (PDB ID 4J7C) and TrkH (PDB ID 3PJZ) were used. The N-terminus of OsHKT2;2 (residues 1-38) was modeled on the basis of TrkH, since the crystal structure of KtrB does not contain the N-terminal region. OsHKT2;2 contains a 29 amino acid long extracellular loop that is not present in bacterial channels. This loop spanning residues 473-501 has been 
modeled separately using the FALC-Loop server, which combines statistical and knowledgebased methods (Ko et al., 2011). The improved full-length model was refined using i3Drefine (Bhattacharya and Cheng, 2013) and minimized using CHARMM via its web portal CHARMMing (Brooks et al., 2009; Miller et al., 2008).

The refined model was embedded in a pre-equilibrated POPC bilayer solvated with preequilibrated TIP3P water molecules (Jorgensen et al., 1983) in a periodic boundary condition box using VMD (Humphrey et al., 1996). The system was neutralized by the addition of sixteen chloride ions. Each, six $\mathrm{Na}^{+}$and $\mathrm{K}^{+}$ions as well as twelve further chloride ions were added to mimic the concentration of $10 \mathrm{mM} \mathrm{NaCl}$ and $10 \mathrm{mM} \mathrm{KCl}$. The entire system underwent several minimization steps and was equilibrated with successive restraint reduction until restraints were removed entirely. Three replicates of 100ns molecular dynamics (MD) simulations were generated using NAMD (Kalé et al., 1999; Phillips et al., 2005).

Binding pocket residues were identified by counting the frames in which an ion was within $4 \AA$ of a given residue during MD simulations. Ion coordination was examined by measuring distances between charged atoms of P71, D75, D501, K504 and the ion present in the binding pocket. Salt bridges were evaluated using VMD's Salt Bridge plugin. Electrostatic potentials were calculated using the APBS web server with the ionic strength set to simulate $10 \mathrm{mM} \mathrm{NaCl}$ and $10 \mathrm{mM} \mathrm{KCl}$ (Unni et al., 2011). APBS input files were generated using the PDB2PQR Server version 2.1.1 (Dolinsky et al., 2004; 2007).

In silico mutations of $O s \mathrm{HKT} 2 ; 2$ were generated via VMD's Mutate plugin. Mutants were minimized and equilibrated followed by $15 \mathrm{~ns}$ of MD simulation in NAMD.

\subsection{Electrophysiology}

Wild-type and mutant $O s \mathrm{HKT} 2 ; 2$ constructs were cloned into the pNB1u vector for expression in Xenopus oocytes using the USER-cloning technique (Nour-Eldin et al., 2006). Utilized primers were: GGCTTAAUEatgacgagcatttaccaagaa (forward) and GGTTTAAU ctaccatagcctccaatatt (reverse). Vector-specific sequences are given in uppercase format, $O s \mathrm{HK}$ T2;2-specific sequences in lowercase format. The position of uracil is underlined. Standard fusion PCR technique was used for site-directed mutagenesis. After DNA linearization with NotI, cRNA was synthesised using the mMessage mMachine in vitro transcription kit following the manufacturer's guidelines.

For expression in oocytes, stage V and VI Xenopus leavis oocytes were harvested and kept in ND96 solution $\left(96 \mathrm{mM} \mathrm{NaCl}, 2 \mathrm{mM} \mathrm{KCl}, 1.8 \mathrm{mM} \mathrm{CaCl}_{2}, 1 \mathrm{mM} \mathrm{MgCl}_{2}, 2.5 \mathrm{mM} \mathrm{Na}\right.$ Pyruvate, $5 \mathrm{mM}$ Hepes $-\mathrm{pH} 7.5,50 \mathrm{mg} \mathrm{mL}^{-1}$ gentamycin and $\left.0.4 \mathrm{~g} \mathrm{~L}^{-1} \mathrm{BSA}\right)$. Oocytes were defolliculated by collagenase treatment in ND96 without $\mathrm{CaCl}_{2}$, gentamycin and $\mathrm{BSA}$ and kept overnight at $18^{\circ} \mathrm{C}$ in complete ND96 solution as described initially. All animal procedures were performed in accordance with Cornell University IACUC Protocol number 2017-0139. 50nl of cRNA $(500 \mathrm{ng} / \mu \mathrm{l})$ were microinjected into oocytes using an oil-driven injection system (Nanoject II Auto-Nanoliter Injector, Drummond Scientific Company, US). Cells were then incubated for two days at $18^{\circ} \mathrm{C}$ in complete ND96 solution. Whole-cell currents were recorded using conventional Two-Electrode Voltage-Clamp technique (GeneClamp 500 amplifier and Digidata 1320APClamp 10 data acquisition system, Axon Instruments). Recordings were carried out under constant perfusion of bath solutions containing $2 \mathrm{mM} \mathrm{MgCl}_{2}, 1.8 \mathrm{mM} \mathrm{CaCl}_{2}, 10 \mathrm{mM} \mathrm{MES}$, pH 5.5 with Tris-Base with the addition of: $\mathrm{Na} 0 \mathrm{~K} 1: 1 \mathrm{mM} \mathrm{KCl}, 165 \mathrm{mM}$ Sorbitol; Na0K30: $30 \mathrm{mM} \mathrm{KCl}$, 130mM Sorbitol; Na03K0: 0.3mM NaCl, 165mM Sorbitol; Na03K1: 0.3mM NaCl, $1 \mathrm{mM} \mathrm{KCl,}$ 165mM Sorbitol; Na30K0: 30mM NaCl, 130mM Sorbitol; Na30K1: 30mM NaCl, 1mM KCl, 
Cation binding pocket of OsHKT2;2

130mM Sorbitol; Na30K30: 30mM NaCl, 30mM KCl, 100mM Sorbitol.

\section{Results}

\subsection{Electrophysiological characterization of OsHKT2;2}

OsHKT2;2 is one out of eight to nine HKT channels (dependent on the cultivar) expressed in rice plants (Garciadeblás et al., 2003). Members of the HKT family show remarkable functional diversity regarding ion selectivity, rectification properties and effect of external cation compositions (Jabnoune et al., 2009). To provide a solid basis for this study, we first characterized the functional properties of OsHKT2;2 expressed in Xenopus leavis oocytes using the Two-Electrode Voltage-Clamp (TEVC) technique.

Under voltage clamp conditions, cells expressing OsHKT2;2 conducted mainly $\mathrm{Na}^{+}$inward currents in the absence of $\mathrm{K}^{+}$. Thereby, ion transport rates raised with increasing $\mathrm{Na}^{+}$ concentrations (Figure 1B, D, I). At low extracellular $\mathrm{Na}^{+}$concentrations $(0.3 \mathrm{mM} \mathrm{NaCl})$, ion currents of several hundred nanoamperes were detected, while at high $\mathrm{Na}^{+}$concentrations $(30 \mathrm{mM} \mathrm{NaCl})$ currents increased to several microamperes consistent with $\mathrm{Na}^{+}$being the ion carrying the current. A 100 -fold increment of $\mathrm{Na}^{+}$concentration (from $0.3 \mathrm{mM}$ to $30 \mathrm{mM}$ ) resulted in an almost 5 -fold increase of ion conduction.

The addition of low $\mathrm{K}^{+}$concentrations $(1 \mathrm{mM} \mathrm{KCl})$ had a stimulating effect on the ion transport and led to a significant increase in ion conduction (Figure 1D, E). One millimolar of $\mathrm{KCl}$ almost doubled the ion conduction at high and low $\mathrm{Na}^{+}$concentrations. We are assuming a stimulating effect of $\mathrm{K}^{+}$ions for two reasons: (1) In the absence of $\mathrm{NaCl}$ in the bath solution, no inward currents could be measured in the presence of 1 or $30 \mathrm{mM} \mathrm{KCl}(\mathrm{Na} 0 \mathrm{~K} 1, \mathrm{Na} 0 \mathrm{~K} 30)$. Currents at negative voltages were comparable to measurements in control oocytes (compare Figure 1G, H, I and Supplemental Figure 3). Therefore, potassium ions, at least in the absence of sodium ions, do not pass the conduction pathway. Hence, it is unlikely that the presence of $1 \mathrm{mM} \mathrm{KCl}$ is simply adding to the current of $30 \mathrm{mM} \mathrm{NaCl}$. (2) This stimulating effect was revoked under rising $\mathrm{K}^{+}$ concentration $(30 \mathrm{mM} \mathrm{KCl}$; Figure $1 \mathrm{~F}, \mathrm{I}, \mathrm{J})$. Interestingly, the ionic current at $30 \mathrm{mM} \mathrm{KCl}$ was comparable to that observed without $\mathrm{K}^{+}$ions in the extracellular solution indicating that $\mathrm{K}^{+}$ stimulates ion conduction only at low concentrations.

Additionally, under high $\mathrm{Na}^{+}$concentrations, inward currents partially deactivated at negative voltages. This effect was most pronounced in the absence of $\mathrm{K}^{+}$and reduced with increasing $\mathrm{K}^{+}$ concentrations (Figure 1D-F, J). At $30 \mathrm{mM} \mathrm{NaCl}$ (no $\mathrm{KCl}$ ), the current amplitude was reduced by $12.0 \%( \pm 2.8 \%)$ over one-second voltage pulses. In the presence of $1 \mathrm{mM} \mathrm{KCl}$, the current reduction was $10.7 \%( \pm 4.6 \%)$, and at $30 \mathrm{mM} \mathrm{KCl}$ current deactivation was almost abolished $(3.2 \% \pm 2.2 \%)$.

Overall, we identified and characterized three functional properties for wild-type $O s \mathrm{HKT} 2 ; 2$ which we used later in the study to compare functional changes in mutated $O s \mathrm{HKT} 2 ; 2$ channels (Table 1). The functional properties of wild-type OsHKT2;2 include: (1) ionic currents enhance with increasing $\mathrm{Na}^{+}$concentration, (2) low $\mathrm{K}^{+}$concentration $(1 \mathrm{mM} \mathrm{KCl})$ stimulates $\mathrm{Na}^{+}$ion transport, and (3) high $\mathrm{K}^{+}$concentration $(30 \mathrm{mM} \mathrm{KCl})$ lacks the stimulating effect on $\mathrm{Na}^{+}$ion transport.

\subsection{Identification of an extracellular cation binding pocket}

In order to identify amino acids that are approached by ions before entering the pore, we built a homology model of the OsHKT2;2 wild-type channel on the basis of the structurally and 
functionally related bacterial channels KtrB (PDB ID 4J7C) and TrkH (PDB ID 3PJZ). Because the N-terminus of KtrB is not included in the crystal structure, N-terminal amino acids of OsHKT2;2 were modeled on the basis of the TrkH (see Methods for details). Subsequently, we performed molecular dynamics (MD) simulation in the presence of $10 \mathrm{mM} \mathrm{NaCl}$ and $10 \mathrm{mM} \mathrm{KCl}$ for $100 \mathrm{~ns}$. This allows the evaluation of the frequency at which ions approach residues over time. To demonstrate reproducibility, the MD simulation was performed threefold and a total of $300 \mathrm{~ns}$ simulation time was reached, which form the basis of the following results.

Analyses of the contact frequency between ions and amino acids during MD simulations led to the identification of a potential extracellular cation binding pocket. This binding pocket is located in the outer extracellular protein region approximately $20 \AA$ away from the pore entrance (Figure 2A). We identified three polar residues and one proline to be involved in the formation of a putative cation binding pocket - P71, D75, D501 and K504. Within these, three are highly conserved among plant HKT channels (P71, D75 and K504), while at position of D501 a negative charge is preserved in $75 \%$ of HKT channel sequences. Figure $2 \mathrm{~B}$ illustrates the conservation of these four residues within the rice HKT family and additionally states their conservation within a multiple sequence alignment based on 20 experimentally characterized HKT channels.

\subsubsection{Structural attributes of the extracellular cation binding pocket}

Both, sodium and potassium ions, approached a defined area on the protein surface during MD simulations. $\mathrm{K}^{+}$ions were found in this region between 0.5 to $1 \%$ of the simulation time, while $\mathrm{Na}^{+}$stayed there about three times longer (1.2 to $5.2 \%$ of the time; Figure $\left.3 \mathrm{~A}\right)$. At this point, it is important to clarify that we were not searching for a region in the sense of an ion binding site that binds ions for extended time periods with potential allosteric impacts on protein function. Instead, we were searching for areas, which ions approach and pass on their way into the pore in other words, binding sites that attract cations and guide them to the pore entrance. That implicates that the total time of residence of an ion within the identified region is comparatively short as observed in our MD simulations. Nevertheless, cations approach the site frequently and are coordinated by the identified residues, in contrast, to randomly approached residues where no ion coordination occurs. Throughout the remaining of the study, we will refer to this region as extracellular cation binding pocket.

Despite their short residence in the binding pocket, the cations are coordinated by surrounding amino acids. A closer examination revealed four oxygen atoms coordinating the cations in this region: the backbone oxygen of P71 (Figure 3B, C.1), one side chain oxygen atom of D75 (Figure 3B, C.2) and the two side chain oxygen atoms of D501 (Figure 3B, C.4 and C.5). The average distances between the mentioned oxygen atoms and $\mathrm{Na}^{+}$were $2.38 \AA, 2.29 \AA, 2.90 \AA$ and $2.58 \AA$, respectively, and, $2.74 \AA, 2.63 \AA, 2.92 \AA$ and $2.90 \AA$ for $\mathrm{K}^{+}$(Figure $3 \mathrm{~B}$ ). The coordination of one $\mathrm{Na}^{+}$ion over time is exemplarily shown in Figure 3C. Besides the proximity $(<2.5 \AA)$ of the $\mathrm{Na}^{+}$ion to the four mentioned oxygen atoms (plots C.1, C.2, C.4, C.5), the proximity to other side chain atoms facing towards the predicted binding pocket is also shown. Namely, the proximity to the second side chain oxygen atom of D75 (plot C.3) and the positively charged nitrogen atom of K504 (plot C.6). The second side chain oxygen atom of D75 is oriented away from the binding pocket most of the time and cannot contribute to the coordination of cations. As expected, the nitrogen of lysine 504 is also not involved in the coordination, since the positive charges of cations and the nitrogen atom repel each other. 
Nevertheless, lysine 504 is located between the two aspartic acids D75 and D501, which suggests an interaction between these residues. Salt bridges may be formed between oppositely charged residues when the charged atoms are closer than $4 \AA$ (Kumar and Nussinov, 2002). Average distances between the positively charged nitrogen atom of lysine 504 and the negatively charged oxygen atoms of both aspartates fulfill this requirement (Figure 4A, B). Indeed, both oxygen atoms of D75 are in constant proximity to the nitrogen of lysine 504 (Figure 4B, upper two traces) with an average distance of $2.83 \AA$ and $2.82 \AA$, respectively (Figure 4C). The two oxygen atoms of D501 alternate in their proximity to this nitrogen (Figure 4B, lower two traces). If in proximity, the distance is $2.68 \AA$ on average (Figure 4C). Thus, lysine 504 may interact with both aspartates and acts as a structural component to obtain the geometry of the binding pocket by keeping the aspartates in place rather than coordinating ions.

Overall, our computational studies suggest that the extracellular cation binding pocket is constituted by a positively charged lysine (K504) that holds two negatively charged aspartates (D75 and D501) in place, which form a negative environment that attracts cations. The side chain oxygen atoms of both aspartates, in conjunction with the backbone oxygen of P71 coordinate $\mathrm{Na}^{+}$and $\mathrm{K}^{+}$ions.

\subsection{Experimental validation of the predicted cation binding pocket}

To study the relevance of the cation binding pocket for the ion conduction of $O s \mathrm{HKT} 2 ; 2$, we mutated P71, D75, D501 and K504 and experimentally characterized the changes in functionality in these mutants. Alanine mutations of all four residues were performed to assess the effect of side chain removal, and asparagine mutants of D75 and D501 allowed the evaluation of negative charges neutralization. Besides, K504 was mutated to an arginine to examine the relevance of the side chain size, to glutamine to analyze the effect of charge neutralization, and to glutamic acid to evaluate the inversion from a positive to a negative charge. Overall, mutation of residue K504 had the most significant impact on OsHKT2;2's functionality. Modifications of D75 and D501 affected the kinetic features slightly, whereas mutation of P71 did not cause changes in the transport mechanism as compared to the wild-type channel (Table 1).

\subsubsection{Mutations of P71, D75 and D501 show no or minor functional alterations}

To evaluate the effect of mutations of the cation coordinating residues we modified P71, D75 and D501 by the removal of side chains (alanine mutants) and charges (asparagine mutants). The mutant P71A behaved similarly to the wild-type channel regarding the three functional properties that have been established before on the basis of wild-type OsHKT2;2: $\mathrm{Na}^{+}$conductivity (1), the mutual ion-species effect at low (2) and high (3) $\mathrm{K}^{+}$concentrations (Table 1). P71A transported $\mathrm{Na}^{+}$in the absence of $\mathrm{K}^{+}$, and the ion transport increased at high $\mathrm{Na}^{+}$concentration. As observed in the wild-type channel, ion currents increased in the presence of $1 \mathrm{mM} \mathrm{K}^{+}$but not $30 \mathrm{mM} \mathrm{K}^{+}$, when compared to currents recorded in the absence of $\mathrm{K}^{+}$(Figure 5).

Mutations of residues D75 and D501 affected the ion conduction slightly. As observed in the wild-type channel, D75A/N and D501A/N transported $\mathrm{Na}^{+}$in the absence of $\mathrm{K}^{+}$and a concentration-dependent manner. However, the increase of the current amplitude was slightly lower for D75A and D501A (4-fold), and only 3-fold in the D501N mutant (Figure 6). The strong stimulating effect of low $\mathrm{K}^{+}$concentrations on the ion conduction was observed in all mutants. However, in contrast to wild-type $O s \mathrm{HKT} 2 ; 2$, low magnitudes of current activation at $30 \mathrm{mM} \mathrm{NaCl}$ were still observed in D75N and D501N mutants (Figure 6C, F, G and Supporting Figure 1). Indicating that the mutual effect of $\mathrm{K}^{+}$on $\mathrm{Na}^{+}$currents had slightly shifted. 
Cation binding pocket of OsHKT2;2

In summary, residues D75 and D501 were more sensitive towards modifications than P71, which was not affected by mutation. The latter was expected since proline coordinates cations via its backbone oxygen atom, which is not altered by mutations.

\subsubsection{K504 mutants showed substantial differences in their functionality compared to wild- type OsHKT2;2}

Lysine 504 was identified as a structural component of the cation binding pocket that seems to take a crucial position in the overall geometry. Indeed, K504 was very sensitive towards residue substitutions. Removal of the side chain (mutant K504A) and inversion of the charge (mutant K504E) rendered $O s \mathrm{HKT} 2 ; 2$ non-functional. K504A and K504E injected oocytes showed no currents two days after cRNA injection (Figure 7E, F). Each, fifteen injected oocytes have been analyzed on three different measuring days. However, oocytes injected with K504A cRNA sporadically conducted low currents in the nanoampere range, which differed significantly from those recorded in control oocytes. These observations were occasionally made three days after cRNA injection. We speculated that the K504A mutant may be functional but conducts ions with extremely low efficiency. In this case, the increased protein expression over time may lead to increased whole-cell currents through OsHKT2;2-K504A that are only detectable after long incubation times.

In contrast, lysine mutants K504R and K504Q were functional although with substantial differences in their transport characteristics as compared to wild-type OsHKT2;2 and all other mutants. $\mathrm{Na}^{+}$currents in the absence of $\mathrm{K}^{+}$were low even at high $\mathrm{Na}^{+}$concentrations $(30 \mathrm{mM}$ $\mathrm{NaCl}$ ). In fact, the current amplitude rose not more than 2-fold with increasing $\mathrm{Na}^{+}$concentration (compared to the 5-fold increase in wild-type channels) and remained in the nanoampere range. Therefore, the increase of external $\mathrm{Na}^{+}$concentration cannot enhance the ion conduction in the same extent as in wild-type OsHKT2;2 (Figure 7B, D, H, J). Nevertheless, low $\mathrm{K}^{+}$ concentrations did maintain their strong stimulating effect on the ion conduction (Figure 7C, I). This effect was even more pronounced in the lysine mutants. In the background of $30 \mathrm{mM} \mathrm{NaCl}$, the addition of $1 \mathrm{mM} \mathrm{KCl}$ resulted in a more than 4- (K504Q) to 6-fold (K504R) increase of the current amplitude. Furthermore, the increase of $\mathrm{K}^{+}$concentration in the extracellular bath media had still a substantial stimulating effect on the ionic conduction. Currents measured at $30 \mathrm{mM}$ $\mathrm{Na}^{+}$and $\mathrm{K}^{+}$were about twice as large in amplitude compared to currents in the presence of $30 \mathrm{mM} \mathrm{Na}^{+}$only (Figure 7D, J). This observation indicates that the $\mathrm{Na}^{+}$transportability of OsHKT2;2 is affected in the K504R and K504Q mutants, while $\mathrm{K}^{+}$ions are still able to promote and, in these mutants, also to rescue proper ion conduction.

\subsection{In silico mutations support experimental results}

To gain further understanding of the nature of the experimental results, the four lysine mutants were studied in silico by performing 100ns MD simulations. In each simulation, we examined the time $\mathrm{Na}^{+}$and $\mathrm{K}^{+}$ions approached one of the four binding pocket residues $(<4 \AA)$ and the total number of approaches throughout the MD simulation (Table 2). The combined assessment of duration of stay and number of approaches allows the evaluation of the average time an ion spent in the cation binding pocket. It is striking that $\mathrm{Na}^{+}$ions approached the binding pocket of the non-functional lysine mutants K504A and K504E more frequently than in the functional mutants K504Q and K504R (35 and 85 times versus 15 and 16 times; Table 2). The approaches observed in the mutants $\mathrm{K} 504 \mathrm{Q} / \mathrm{R}$ were comparable to the number of $\mathrm{Na}^{+}$ion approaches in wild-type OsHKT2;2. 
In $O s \mathrm{HKT} 2 ; 2-\mathrm{K} 504 \mathrm{R}$, where the positive charge was kept but the side chain slightly increased, $\mathrm{Na}^{+}$, as well as $\mathrm{K}^{+}$, approached the binding pocket only shortly (see the short duration of stay and the low number of approaches in Table 2). Both cations were most of the time close to residue D501 $\left(\mathrm{Na}^{+}: 2.5 \%\right.$ and $\mathrm{K}^{+}: 3.2 \%$ of simulation time), while in the wild type the duration of stay was more balanced between the four binding pocket residues. It is plausible that a more voluminous arginine residue at position 504 is preventing cations from entering into the binding pocket due to the repulsion of positive charges. Although, the surface area around the binding pocket is still negatively charged and able to attract cations, the positive charge at position 504 is in the case of the mutant bigger and further exposed to the extracellular space than in the wildtype channel, which may cause repulsion of cations (Figure 8E, J compared to A, F).

In mutant K504Q, where lysine's charge was neutralized, cations stayed at least five times longer in the binding pocket $\left(12.9 \%\right.$ and $26.8 \%$ for $\mathrm{Na}^{+}$and $\mathrm{K}^{+}$, respectively) than in mutant $\mathrm{K} 504 \mathrm{R}$, although both mutants showed comparable ion transport abilities in electrophysiological experiments. In contrast to mutant K504R, cations entered into the binding pocket in mutant K504Q, which may account for the more extended stay in the pocket (compare Figure 8I, J). Additionally, $\mathrm{K}^{+}$ions approached the binding pocket six times more frequently than in K504R, which may account at least in part for the longer duration of stay of $\mathrm{K}^{+}$in the binding pocket. Compared to the wild type, cations stayed significantly longer close to the binding pocket. A similar increase (at least for $\mathrm{Na}^{+}$ions) was observed in mutant K504A. The absence of the positive charge may allow cations to stay longer in the negatively charged binding pocket before continuing to the pore entrance of the channel.

In the non-functional mutants K504A and K504E, $\mathrm{Na}^{+}$approached the binding pocket with high frequency (35 and 85 times) and stayed up to $9.6 \%$ (K504A) and $27.4 \%$ (K594E) of the simulation time. $\mathrm{K}^{+}$ions stayed shorter time than $\mathrm{Na}^{+}$ions in the binding pocket in mutant K504A (up to 7.3\%) and longer in mutant K504E (up to 38.7\%). The insertion of a negatively charged glutamate in mutant K504E generated a large negative area on the protein surface compared to OsHKT2;2-wt (Figure 7C compared to A). A negative electrostatic potential highly attracts cations, and more than one cation approached the ion binding pocket at a time (Figure 7G). The long duration of stay indicates that the ions get trapped in the binding pocket, which may account for the absence of ion conduction in $O s \mathrm{HKT} 2 ; 2-\mathrm{K} 504 \mathrm{E}$.

In summary, in silico data provide insights into potential reasons for functional alterations and the non-functioning of K504 mutants that are in good agreement with experimental data.

\section{Discussion}

HKT channels have been described as crucial players in salt tolerance. They transport $\mathrm{Na}^{+}$as uniporter (class I) or in symport with $\mathrm{K}^{+}$ions (class II). Class II-type HKT channels function as $\mathrm{Na}^{+}$uniporter in the absence of $\mathrm{K}^{+}$. However, they become $\mathrm{Na}^{+} / \mathrm{K}^{+}$symporter in the presence of $\mathrm{K}^{+}$. This mutual effect of ion-species on the ion conduction has been observed for several HKT channels. Dependent on the channel member the influence on ion transport varies (Jabnoune et al., 2009). Our electrophysiological studies confirmed the stimulating effect that $\mathrm{K}^{+}$ions exert on $\mathrm{Na}^{+}$transport in $O s \mathrm{HKT} 2 ; 2$. Interestingly, this effect was only seen at low $\mathrm{K}^{+}$concentrations and decreased as the extracellular $\mathrm{K}^{+}$concentration increased. This phenomenon has been previously reported for $O s H K T 2 ; 2$ (Oomen et al., 2012). From the physiological point of view, this is consistent behavior. During $\mathrm{K}^{+}$starvation (low $\mathrm{K}^{+}$concentrations), ion uptake increases and with it the uptake of $\mathrm{Na}^{+}$ions. Physico-chemically similar $\mathrm{Na}^{+}$ions may complement functions of $\mathrm{K}^{+}$ 
up to a certain degree and may enable the survival of the plant while $\mathrm{K}^{+}$availability is low. With increasing concentration of $\mathrm{K}^{+}$, the ion conduction is reduced and with it the uptake of $\mathrm{Na}^{+}$ions. In the presence of sufficient $\mathrm{K}^{+}$ions, high $\mathrm{Na}^{+}$influx is not desired since $\mathrm{Na}^{+}$ions compete with $\mathrm{K}^{+}$and may cause symptoms of $\mathrm{K}^{+}$starvation. However, while the physiological response of HKT is well investigated, the ion-specific effect at the molecular level is still unknown.

To get deeper insights into the molecular basis behind the mutual ion-species effect, we modeled the structure of $\mathrm{OsHKT}_{\mathrm{s}} ; 2$ and analyzed cation-protein contacts. Our computational studies show that four residues P71, D75, D501 and K504 form an extracellular cation binding pocket. Thereby, P71, D75 and D501 coordinate cations and the positively charged lysine (K504) holds the two negatively charged aspartates, D75 and D501, in place. Thus, a negative environment that attracts cations is formed.

We confirmed the relevance of the binding pocket for the ion conduction of $O s \mathrm{HKT} 2 ; 2$ in functional electrophysiological studies. Out of the three residues involved in ion coordination, residue D501 is slightly more sensitive towards modifications. D75 and P71 are located nearby on one extracellular end of a transmembrane helix and contribute to the cation coordination with each one oxygen atom. D501 is placed on the opposite side on the extracellular end of another helix and coordinates with two oxygen atoms. Therefore, mutating D501 removes all coordinating atoms from this side of the binding pocket, while mutating D75 is only eliminating one of two coordinating atoms from the opposite side, which may account for the slightly stronger effect seen in D501 mutants. In either case, these mutations maintain the overall functionality of $O_{s} \mathrm{HKT} 2 ; 2$ and affect its kinetics only slightly. Considering this binding pocket as one of many regions that ions pass before entering the pore, it is plausible that mutations affect the channel kinetics instead of rendering the channel non-functional. The identified binding pocket would be involved in the attraction of cations and their forwarding towards the pore entrance, rather than in the tight and constant binding of ions. Therefore, modifying the coordinating oxygen atoms would first affect transport dynamics.

Lysine 504, on the other hand, is a crucial residue in the binding pocket. Inverting the charge from positive to negative by a glutamate substitution results in a non-conductive mutant. With a third negative charge in the binding pocket, the two aspartates repulse and drift apart, which leads to an expansion of the binding pocket. Additionally, the three negative charges form a large negative region on the protein surface that attracts cations and traps them in the binding pocket. If the identified binding pocket is understood as one stopover for ions on their way into the pore, the trapped ions may explain the inhibited ion conduction, since they might block the path for ions leading into the pore.

Overall, the positively charged lysine 504 may be the necessary impulse that cations need to be forwarded towards the pore. The positive charge and moreover its correct size and position are crucial for channel functionality. The sensitivity towards changes of one of the parameters size or charge indicates how fine-tuned the constitution of the binding pocket is. Removing the side chain by mutations to alanine renders $O s \mathrm{HKT} 2 ; 2$ seemingly non-functional. Neutralizing the positive charge (K504Q) or increasing the size of the amino acid side chain with simultaneous maintenance of its charge (K504R), led to substantial changes in the conductance of OsHKT2;2. In the latter, the $\mathrm{Na}^{+}$uniport is strongly reduced at high $\mathrm{Na}^{+}$concentrations. However, at low $\mathrm{K}^{+}$ concentrations, these mutants still mediate large ion currents indicating that either the binding pocket still works correctly for $\mathrm{K}^{+}$ions or that the positive effect of $\mathrm{K}^{+}$ions has another origin. One possibility may be, e.g. the existence of another ion binding pocket that is more relevant for the $\mathrm{K}^{+}$forwarding than the one described here. In fact, during simulations, we could identify 
additional regions that are approached by ions on their way into the pore. These regions are currently under investigation.

The importance of K504 has been shown earlier for the wheat TaHKT1 channel (Kato et al., 2007). In this study, the mutant K508Q, which corresponds to K504Q in our present study, showed reduced functionality in comparison to the wild-type channel in electrophysiological experiments and yeast complementation studies. The authors also proposed a potential salt bridge formation between K508 and D78, which corresponds to D75 in OsHKT2;2. Those results and the ones presented in our study point to the functional relevance of K504 in combination with D75. Our study additionally provides a biological and structural context by identifying these residues as part of a cation binding pocket.

Overall, using computational methods, we identified an extracellular cation binding pocket and validated its functional relevance experimentally. Although being about $20 \AA$ far from the pore entrance, the identified binding pocket is essential for proper ion conduction. Essential ion binding pockets on the extracellular protein surface provide an example of how different ion species may affect the channel conduction behavior even before entering the pore. We are still far from understanding how $\mathrm{K}^{+}$ions influence the ion transport of OsHKT2;2 on the molecular level. This study, however, provides new evidence, which opens the discussion for new explanations for the mutual effect of ion species.

\section{Acknowledgments}

We thank Dr. Julian Schroeder (University of California, San Diego) for providing the clone of OsHKT2;2 wild-type. We also thank Alison Coluccio for technical assistance. This work was supported by grants from the Chilean Fondo Nacional de Desarrollo Científico y Tecnológico (http://www. conicyt.cl/fondecyt) to JR (No. 3150173), AV-J (No. 11170223), ID (No. 1150054) and WG (No. 1140624), and from Conicyt, programme PAI, Convocatoria nacional subvención a la instalación en la academia 2017 to JR (No. PAI77170035).

\section{Conflict of interest}

The authors declare that they have no conflicts of interest with the contents of this article.

\section{Author contributions}

JR conceived and coordinated the study and wrote the paper. JR and AVJ designed, performed and analyzed the computational experiments. JR and MP designed, performed and analyzed the electrophysiological experiments. JR, ID and WG interpreted the data. All authors reviewed the results, revised the manuscript and approved its final version.

\section{References}

Adams, E., and Shin, R. (2014). Transport, signaling, and homeostasis of potassium and sodium in plants. J Integr Plant Biol 56, 231-249. doi:10.1111/jipb.12159.

Almeida, P., Katschnig, D., and de Boer, A. H. (2013). HKT transporters--state of the art. Int J Mol Sci 14, 20359-20385. doi:10.3390/ijms141020359. 
Cation binding pocket of OsHKT2;2

Benito, B., Haro, R., Amtmann, A., Cuin, T. A., and Dreyer, I. (2014). The twins K+ and Na+ in plants. J Plant Physiol 171, 723-731. doi:10.1016/j.jplph.2013.10.014.

Bhattacharya, D., and Cheng, J. (2013). i3Drefine software for protein 3D structure refinement and its assessment in CASP10. PLoS ONE 8, e69648. doi:10.1371/journal.pone.0069648.

Böhm, J., Scherzer, S., Shabala, S., Krol, E., Neher, E., Mueller, T. D., et al. (2015). Venus flytrap HKT1-type channel provides for prey sodium uptake into carnivorous plant without conflicting with electrical excitability. Mol Plant 9, 428-436. doi:10.1016/j.molp.2015.09.017.

Brooks, B. R., Brooks, C. L., Mackerell, A. D., Nilsson, L., Petrella, R. J., Roux, B., et al. (2009). CHARMM: the biomolecular simulation program. J Comput Chem 30, 1545-1614. doi:10.1002/jcc. 21287 .

Dolinsky, T. J., Czodrowski, P., Li, H., Nielsen, J. E., Jensen, J. H., Klebe, G., et al. (2007). PDB2PQR: expanding and upgrading automated preparation of biomolecular structures for molecular simulations. Nucleic Acids Res 35, W522-5. doi:10.1093/nar/gkm276.

Dolinsky, T. J., Nielsen, J. E., McCammon, J. A., and Baker, N. A. (2004). PDB2PQR: an automated pipeline for the setup of Poisson-Boltzmann electrostatics calculations. Nucleic Acids Res 32, W665-7. doi:10.1093/nar/gkh381.

Flowers, T., and Läuchli, A. (1983). Sodium versus potassium: substitution and compartmentation. In Inorganic plant nutrition 15B, 651-681.

Garciadeblás, B., Senn, M. E., Bañuelos, M. A., and Rodríguez-Navarro, A. (2003). Sodium transport and HKT transporters: the rice model. Plant J 34, 788-801.

Gassmann, W., Rubio, F., and Schroeder, J. I. (1996). Alkali cation selectivity of the wheat root high - affinity potassium transporter HKT1. The Plant Journal 10, 869-882.

Haro, R., Bañuelos, M. A., Senn, M. E., Barrero-Gil, J., and Rodríguez-Navarro, A. (2005). HKT1 mediates sodium uniport in roots. Pitfalls in the expression of HKT1 in yeast. Plant Physiol 139, 1495-1506. doi:10.1104/pp.105.067553.

Hasegawa, P. M., Bressan, R. A., Zhu, J.-K., and Bohnert, H. J. (2000). PLANT CELLULAR AND MOLECULAR RESPONSES TO HIGH SALINITY. Annu. Rev. Plant Physiol. Plant Mol. Biol. 51, 463-499. doi:10.1146/annurev.arplant.51.1.463.

Horie, T., Costa, A., Kim, T. H., Han, M. J., Horie, R., Leung, H.-Y., et al. (2007). Rice OsHKT2;1 transporter mediates large $\mathrm{Na}+$ influx component into $\mathrm{K}+$-starved roots for growth. EMBO J 26, 3003-3014. doi:10.1038/sj.emboj.7601732.

Horie, T., Hauser, F., and Schroeder, J. I. (2009). HKT transporter-mediated salinity resistance mechanisms in Arabidopsis and monocot crop plants. Trends Plant Sci 14, 660-668.

Horie, T., Yoshida, K., Nakayama, H., Yamada, K., Oiki, S., and Shinmyo, A. (2001). Two types 
Cation binding pocket of OsHKT2;2

of HKT transporters with different properties of $\mathrm{Na}+$ and $\mathrm{K}+$ transport in Oryza sativa. Plant $J 27,129-138$.

Humphrey, W., Dalke, A., and Schulten, K. (1996). VMD: visual molecular dynamics. J Mol Graph 14, 33-8, 27-8.

Jabnoune, M., Espeout, S., Mieulet, D., Fizames, C., Verdeil, J.-L., Conéjéro, G., et al. (2009). Diversity in expression patterns and functional properties in the rice HKT transporter family. Plant Physiol 150, 1955-1971. doi:10.1104/pp.109.138008.

Jorgensen, W. L., Chandrasekhar, J., Madura, J. D., Impey, R. W., and Klein, M. L. (1983). Comparison of simple potential functions for simulating liquid water. $J$ Chem Phys 79, 926935. doi:10.1063/1.445869.

Kalé, L., Skeel, R., Bhandarkar, M., Brunner, R., Gursoy, A., Krawetz, N., et al. (1999). NAMD2: Greater Scalability for Parallel Molecular Dynamics. Journal of Computational Physics 151, 283-312. doi:10.1006/jcph.1999.6201.

Kato, N., Akai, M., Zulkifli, L., Matsuda, N., Kato, Y., Goshima, S., et al. (2007). Role of positively charged amino acids in the M2D transmembrane helix of Ktr/Trk/HKT type cation transporters. Channels (Austin) 1, 161-171.

Ko, J., Lee, D., Park, H., Coutsias, E. A., Lee, J., and Seok, C. (2011). The FALC-Loop web server for protein loop modeling. Nucleic Acids Res 39, W210-4. doi:10.1093/nar/gkr352.

Kumar, S., and Nussinov, R. (2002). Close-range electrostatic interactions in proteins. Chembiochem 3, 604-617. doi:10.1002/1439-7633(20020703)3:7<604::AIDCBIC604>3.0.CO;2-X.

Landau, M., Mayrose, I., Rosenberg, Y., Glaser, F., Martz, E., Pupko, T., et al. (2005). ConSurf 2005: the projection of evolutionary conservation scores of residues on protein structures. Nucleic Acids Res 33, W299-302. doi:10.1093/nar/gki370.

Leigh, R. A., and Wyn Jones, R. G. (1984). A hypothesis relating critical potassium concentrations for growth to the distribution and functions of this ion in the plant cell. New Phytol 97, 1-13. doi:10.1111/j.1469-8137.1984.tb04103.x.

Maathuis, F. J. M. (2009). Physiological functions of mineral macronutrients. Curr Opin Plant Biol 12, 250-258. doi:10.1016/j.pbi.2009.04.003.

Marschner, P. (2012). Marschner's Mineral Nutrition of Higher Plants. London: Academic Press.

Maser, P., Eckelman, B., Vaidyanathan, R., and Horie, T. (2002). Altered shoot/root Na+ distribution and bifurcating salt sensitivity in Arabidopsis by genetic disruption of the $\mathrm{Na}+$ transporter AtHKT1. FEBS letters 531, 157-161.

Miller, B. T., Singh, R. P., Klauda, J. B., Hodoscek, M., Brooks, B. R., and Woodcock, H. L. 
Cation binding pocket of OsHKT2;2

(2008). CHARMMing: a new, flexible web portal for CHARMM. J Chem Inf Model 48, 1920-1929. doi:10.1021/ci800133b.

Munns, R., James, R. A., Xu, B., Athman, A., Conn, S. J., Jordans, C., et al. (2012). Wheat grain yield on saline soils is improved by an ancestral $\mathrm{Na}+$ transporter gene. Nat. Biotechnol. 30, 360-364. doi:10.1038/nbt.2120.

Møller, I. S., Gilliham, M., Jha, D., Mayo, G. M., Roy, S. J., Coates, J. C., et al. (2009). Shoot $\mathrm{Na}+$ exclusion and increased salinity tolerance engineered by cell type-specific alteration of $\mathrm{Na}+$ transport in Arabidopsis. Plant Cell 21, 2163-2178. doi:10.1105/tpc.108.064568.

Nour-Eldin, H. H., Hansen, B. G., Nørholm, M. H. H., Jensen, J. K., and Halkier, B. A. (2006). Advancing uracil-excision based cloning towards an ideal technique for cloning PCR fragments. Nucleic Acids Res 34, e122-e122. doi:10.1093/nar/gk1635.

Oomen, R. J. F. J., Benito, B., Sentenac, H., Rodríguez-Navarro, A., Talón, M., Véry, A.-A., et al. (2012). HKT2;2/1, a $\mathrm{K}^{+}$-permeable transporter identified in a salt-tolerant rice cultivar through surveys of natural genetic polymorphism. Plant J 71, 750-762. doi:10.1111/j.1365313X.2012.05031.x.

Pardo, J. M., and Quintero, F. J. (2002). Plants and sodium ions: keeping company with the enemy. Genome Biol. 3, reviews 1017.1-1017.4.

Phillips, J. C., Braun, R., Wang, W., Gumbart, J., Tajkhorshid, E., Villa, E., et al. (2005). Scalable molecular dynamics with NAMD. J Comput Chem 26, 1781-1802. doi:10.1002/jcc. 20289 .

Roy, A., Kucukural, A., and Zhang, Y. (2010). I-TASSER: a unified platform for automated protein structure and function prediction. Nat Protoc 5, 725-738. doi:10.1038/nprot.2010.5.

Rubio, F., Gassmann, W., and Schroeder, J. I. (1995). Sodium-driven potassium uptake by the plant potassium transporter HKT1 and mutations conferring salt tolerance. Science 270, 1660-1663.

Su, A., Mager, S., Mayo, S. L., and Lester, H. A. (1996). A multi-substrate single-file model for ion-coupled transporters. Biophys J 70, 762-777. doi:10.1016/S0006-3495(96)79616-9.

Unni, S., Huang, Y., Hanson, R. M., Tobias, M., Krishnan, S., Li, W. W., et al. (2011). Web servers and services for electrostatics calculations with APBS and PDB2PQR. J Comput Chem 32, 1488-1491. doi:10.1002/jcc.21720.

Yao, X., Horie, T., Xue, S., Leung, H.-Y., Katsuhara, M., Brodsky, D. E., et al. (2010). Differential sodium and potassium transport selectivities of the rice OsHKT2;1 and OsHKT2;2 transporters in plant cells. Plant Physiol 152, 341-355.

doi:10.1104/pp.109.145722. 
Cation binding pocket of OsHKT2;2

\section{Tables}

Table 1: Electrophysiological characterisation of OsHKT2;2 and its mutants. The presence of three current characteristics are specified according to their degree of occurrence: very strong $(+++)$, medium $(++)$ or weak $(+)$.

\begin{tabular}{|c|c|c|c|c|}
\hline & Functionality & $\begin{array}{c}\text { Currents enhance at rising } \\
\mathrm{Na}^{+} \text {concentration } \\
(\mathrm{Na03K0} \text { vs. } \mathrm{Na30K0})\end{array}$ & $\begin{array}{c}\text { Current } \\
\text { increase with } \\
1 \mathrm{mM} \mathrm{K}\end{array}$ & $\begin{array}{c}\text { Lack of current } \\
\text { enhancement at rising } \\
\mathrm{K}^{+} \text {concentration } \\
(\mathrm{Na30K0} \text { vs. } \mathrm{Na30K30})\end{array}$ \\
\hline wt & & $\begin{array}{l}+++ \\
++\end{array}$ & $\begin{array}{l}+++ \\
++\end{array}$ & +++ \\
\hline P71A & like wt & +++ & +++ & +++ \\
\hline D75A & comparable to $\mathrm{wt}$ & ++ & +++ & +++ \\
\hline D75N & comparable to wt & +++ & +++ & ++ \\
\hline D501A & comparable to $\mathrm{wt}$ & ++ & +++ & +++ \\
\hline D501N & comparable to $\mathrm{wt}$ & ++ & +++ & ++ \\
\hline K504R & altered kinetics & + & +++ & + \\
\hline K504Q & altered kinetics & + & +++ & + \\
\hline K504A & not functional* & & & \\
\hline K504E & not functional & & & \\
\hline
\end{tabular}

*In some oocytes, very small currents could be detected 2-3 days after RNA injection. This mutant might function in a very inefficient manner so that currents accumulate to a measurable current only after long expression times. After two days of expression though, no currents comparable to wt or other mutants could be measured.

\section{Table 2: Ion approaches of external binding pocket in K504 in silico mutants compared to} OsHKT2;2-wt.

The proportion of time which cations stay within $4 \AA$ of binding pocket forming residues. Time is expressed as a percentage during 100ns MD simulations. In addition, the number of approaches per ion species is indicated.

\begin{tabular}{ccccccccccc}
\hline $\begin{array}{c}\text { (\% of MD) } \\
\text { Residue }\end{array}$ & \multicolumn{2}{c}{ wt } & \multicolumn{2}{c}{ K504R } & \multicolumn{2}{c}{ K504Q } & \multicolumn{2}{c}{ K504A } & \multicolumn{2}{c}{ K504E } \\
& $\mathrm{Na}^{+}$ & $\mathrm{Na}^{+}$ & $\mathrm{K}^{+}$ & $\mathrm{Na}^{+}$ & $\mathrm{K}^{+}$ & $\mathrm{Na}^{+}$ & $\mathrm{K}^{+}$ & $\mathrm{Na}^{+}$ & $\mathrm{K}^{+}$ \\
\hline \hline P71 & 1.9 & 0.4 & 0.5 & 1.4 & 6.0 & 4.4 & 1.9 & 0.3 & 3.2 & 1.0 \\
D75 & 1.4 & 0.2 & 0.1 & 0.6 & 10.0 & 26.8 & 8.4 & 6.9 & 27.4 & 27.7 \\
D501 & 1.8 & 0.5 & 2.5 & 3.2 & 12.9 & 13.8 & 9.6 & 7.3 & 16.0 & 11.6 \\
K504X & 2.3 & 0.2 & 0.2 & 0.4 & 8.0 & 21.2 & 0.02 & - & 24.0 & 38.7 \\
\hline $\begin{array}{c}\text { No. of } \\
\text { approaches }\end{array}$ & 12 & 12 & 16 & 7 & 15 & 43 & 35 & 7 & 85 & 33 \\
\hline
\end{tabular}


Cation binding pocket of OsHKT2;2

Figure legends

Figure 1: Electrophysiological characteristics of OsHKT2;2 in Xenopus oocytes. (A) Representative currents elicited in control cells (not injected with cRNA). Only currents for $\mathrm{Na30K} 1$ solution are shown since highest currents are expected under these conditions. Control currents for all conditions are shown in Supporting Figure 3. (B-H) Representative OsHKT2;2 mediated whole cell currents recorded two days after cRNA injection. Currents were recorded at indicated $\mathrm{Na}^{+}$and $\mathrm{K}^{+}$concentrations: $\mathrm{Na0K} 1-$ no $\mathrm{NaCl}$ and $1 \mathrm{mM} \mathrm{KCl}, \mathrm{Na} 0 \mathrm{~K} 30-$ no $\mathrm{NaCl}$ and $30 \mathrm{mM} \mathrm{KCl}, \mathrm{Na} 03 \mathrm{~K} 0-0.3 \mathrm{mM} \mathrm{NaCl}$ without $\mathrm{KCl}, \mathrm{Na} 03 \mathrm{~K} 1-0.3 \mathrm{mM} \mathrm{NaCl}$ and $1 \mathrm{mM} \mathrm{KCl}$, $\mathrm{Na} 30 \mathrm{~K} 0-30 \mathrm{mM} \mathrm{NaCl}$ without $\mathrm{KCl}$, Na30K1 - 30mM NaCl and $1 \mathrm{mM} \mathrm{KCl,} \mathrm{Na30K30} \mathrm{-} \mathrm{30mM}$ $\mathrm{NaCl}$ and $30 \mathrm{mM} \mathrm{KCl}$. The holding potential was set to the zero current level (approximately $45 \mathrm{mV}$ with solution $\mathrm{Na30K} 1$ ) and 1s voltage pulses were stepped from +20 to $-160 \mathrm{mV}$ in $-15 \mathrm{mV}$ decrements. A $1.5 \mathrm{~s}$ resting interval was allowed between successive voltage steps. The current $(\mu \mathrm{A})$ and time (ms) scale for all traces shown in A through $\mathrm{H}$ is shown at the bottom of panel A. (I) Mean current-voltage (IV) curves. Standard deviation calculated from data obtained from five to eight measurements obtained on five different days and from four different oocyte batches. Currents were normalized to currents measured at $-145 \mathrm{mV}$ in Na30K1 solution. (J) Currents elicited by a $-145 \mathrm{mV}$ voltage pulse, measured at the beginning (dark grey) or end (white) of the voltage pulse, and as average currents over the whole voltage pulse (light grey). Currents were normalized to currents measured in Na30K1 solution. Numbers in the plot indicate the degree of current reduction over time (from beginning to end of the voltage pulse). The magnitude of deactivation is stated in percentage. Means and standard deviation were obtained from data of five to eight measurements.

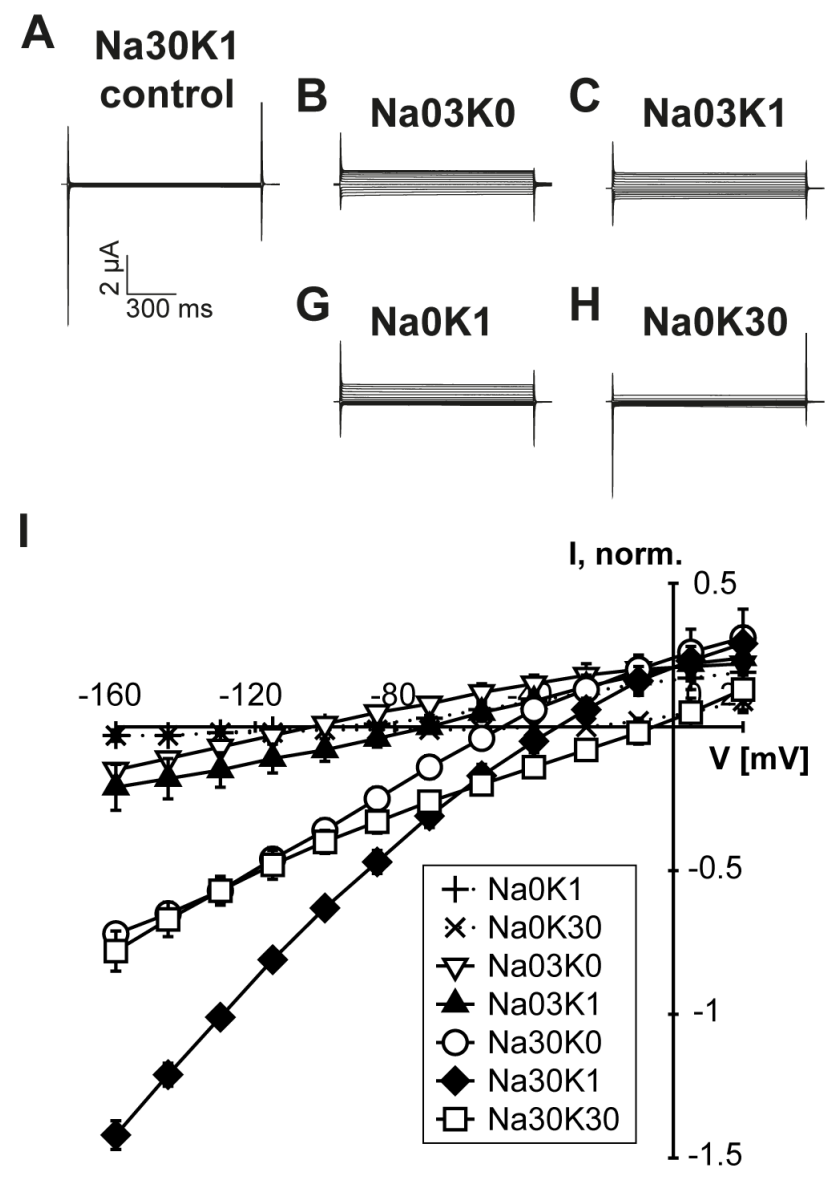

OsHKT2:2-wt
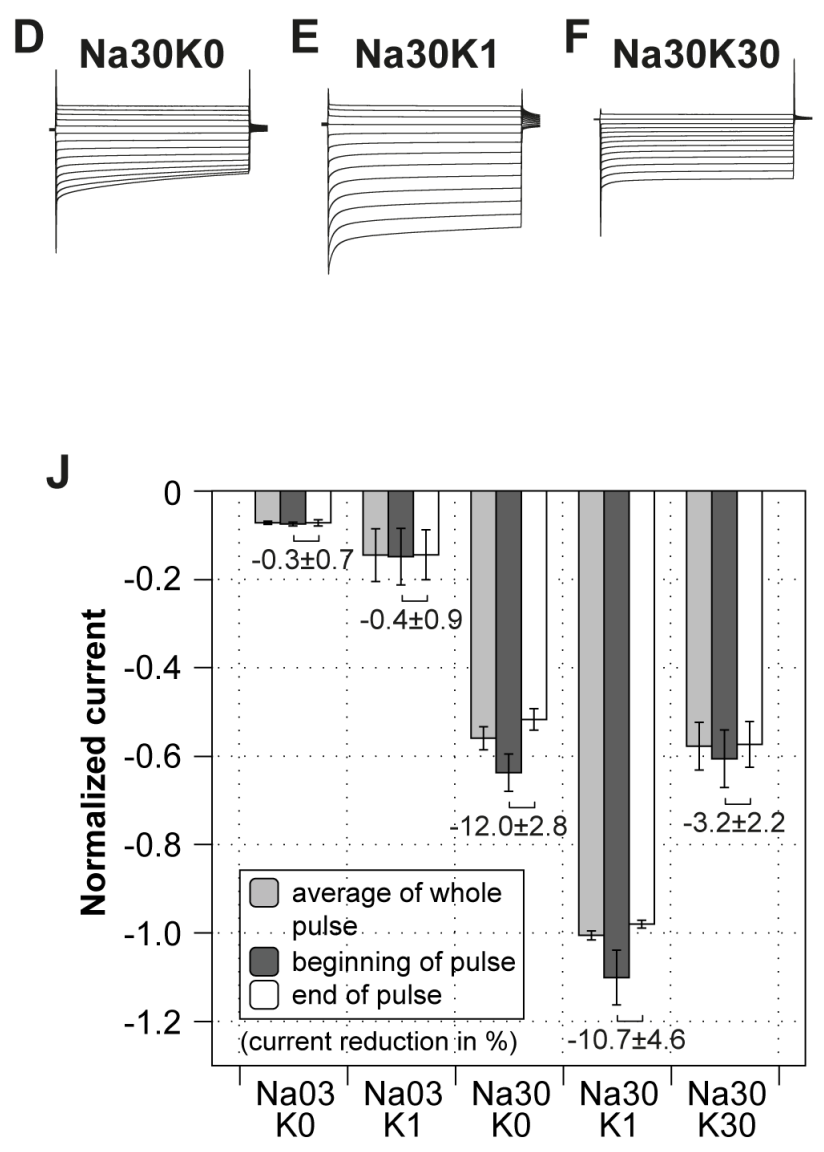
Cation binding pocket of OsHKT2;2

Figure 2: Localization and conservation of extracellular cation binding pocket. (A) OsHKT2;2 is displayed as transparent surface representation with the selectivity filter and surrounding residues marked in the center of the channel as red surface representation. The cation binding pocket is marked on the outer extracellular edge of the protein also as red surface representation, and the position of the four contributing residues is shown. The approximate distance between binding pocket and pore is indicated in the top view representation. (B) An extract of the multiple sequence alignment of all eight HKT members present in Oryza sativa. Conservation of the residues P71, D75, D501 and K504 forming the binding pocket is indicated. ConSurf grades were calculated on the basis of a multiple sequence alignment of 20 experimentally characterized HKT sequences, and are shown below the sequence alignment. The grades range from 1 (variable) to 9 (conserved) (Landau et al., 2005). D75 and K504 are 100\% conserved (ConSurf grade 9). P71 is in 90\% of HKT sequences conserved (ConSurf grade 8), and contains in $5 \%$ of the sequences a lysine or serine. At position 501 a negative charge is somewhat conserved as aspartic in $60 \%$ of the sequences (ConSurf grade 4) or glutamic acid (in $15 \%$ of the sequences). In the remaining $25 \%$ of the sequences, this position holds an asparagine $(10 \%)$, proline $(5 \%)$, lysine $(5 \%)$, or tryptophan $(5 \%)$.

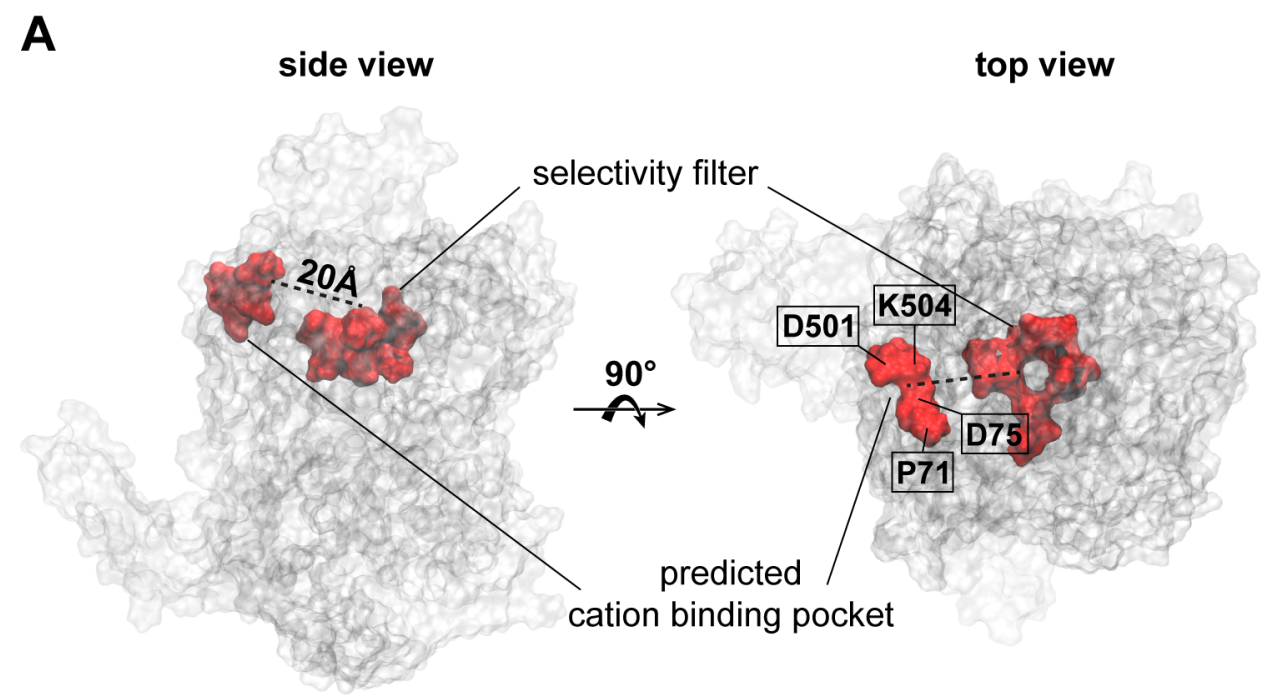

B

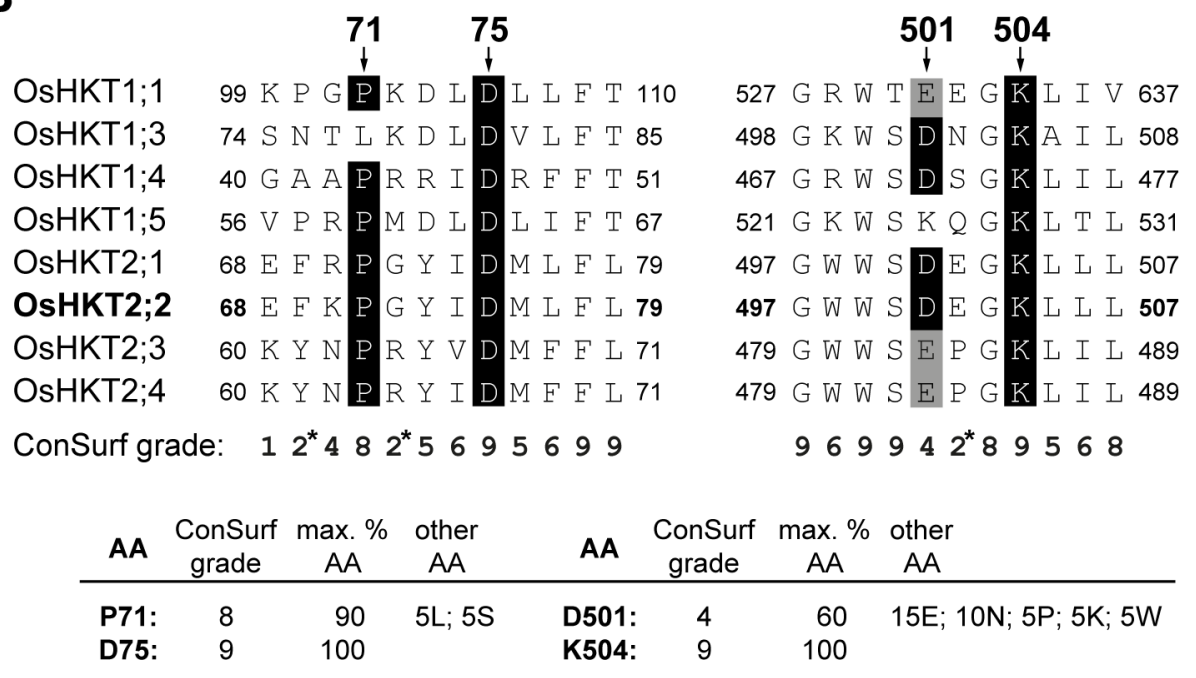


Cation binding pocket of OsHKT2;2

Figure 3: Oxygen atoms of P71, D75 and D501 coordinate cations. (A) Total time that $\mathrm{K}^{+}$and $\mathrm{Na}^{+}$stay in the binding pocket during each of the three simulations expressed in percentage. (B) Coordination of cations happens via the backbone oxygen atom of P71, one side chain oxygen atom of D75 and both side chain oxygen atoms of D501 (red spheres). Average ion-atom distances for sodium and potassium are indicated in the figure. Sodium: 1) $2.38 \pm 0.14 \AA$, 2) $2.29 \pm 0.09 \AA$, 3) $4.02 \pm 0.21 \AA$, 4) $2.90 \pm 0.65 \AA$, 5) $2.58 \pm 0.31 \AA$ and 6) $3.74 \pm 0.25 \AA$. Potassium: 1) $2.74 \pm 0.19 \AA$, 2) $2.63 \pm 0.30 \AA$, 3) $4.15 \pm 0.40 \AA$, 4) $2.92 \pm 0.47 \AA$, 5) $2.90 \pm 0.38 \AA$ and 6) $3.96 \pm 0.28 \AA$. (C) Exemplary plot of ion-atom distances for sodium. Distances of one sodium ion and all six charged atoms facing the potential binding pocket are represented for 5ns of MD system 1 while $\mathrm{Na}^{+}$is inside the pocket. The close proximity $(<2.5 \AA)$ of $\mathrm{Na}^{+}$to the backbone oxygen of P71, one side chain oxygen of D75 and both side chain oxygen atoms of D501 is illustrated, indicating their ability to coordinate sodium.

A

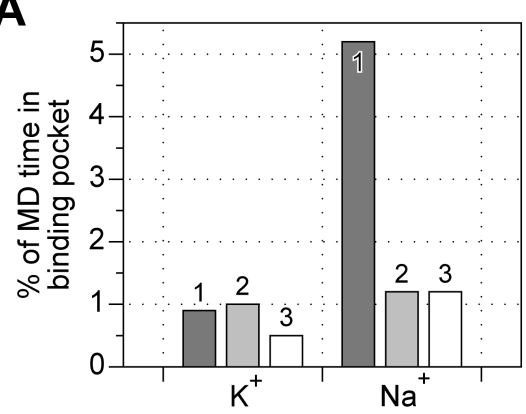

B

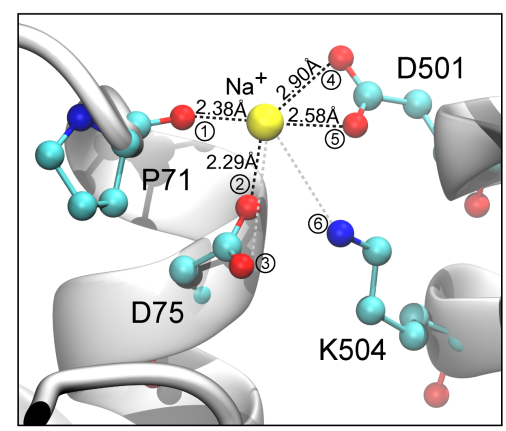

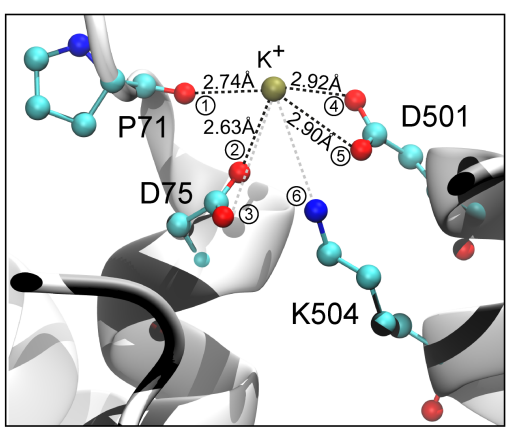

C

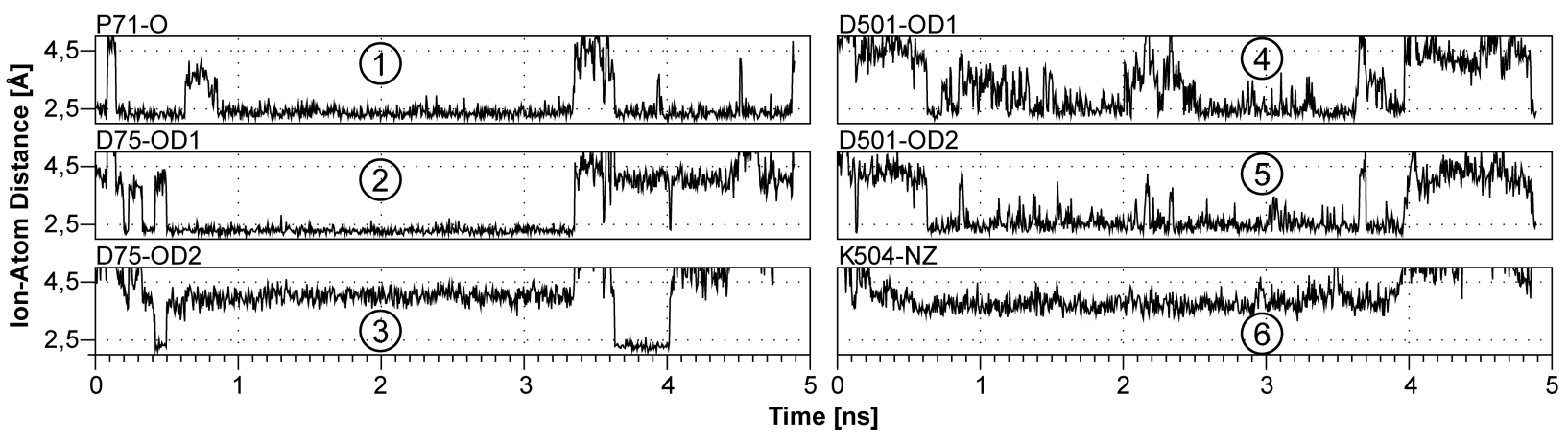


Cation binding pocket of OsHKT2;2

Figure 4: K504 can form salt bridges with D75 and D501. (A) Representation of potential salt bridges between K504 and D75 and D501. The median oxygen-nitrogen distance is stated. (B) Exemplary plot of oxygen-nitrogen distances throughout one complete simulation. The formation of salt bridges is considered possible when oxygen-nitrogen distances are below the threshold of 3.2A. The red line in each plot indicates this threshold. (C) Distance distribution of indicated oxygen-nitrogen distances over all three simulations. The median is stated in each box plot. Outliers are not drawn. For each of the two D501 oxygen atoms only those time spans where the respective oxygen atom was orientated towards K504's nitrogen atom were used.

A

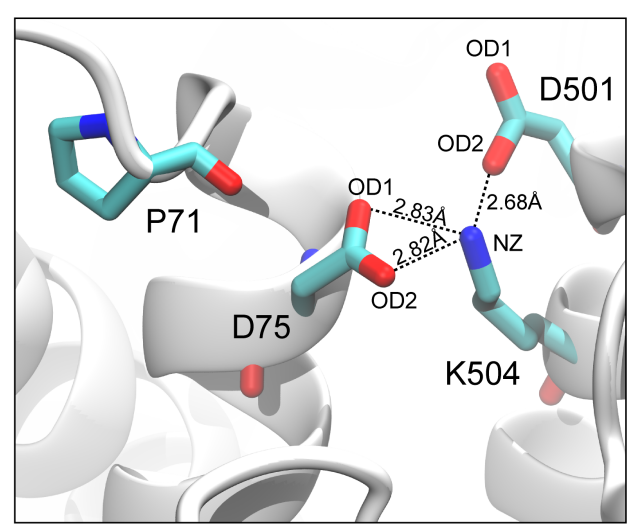

C

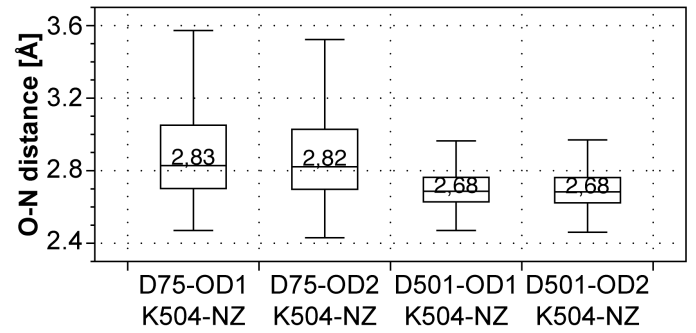

B
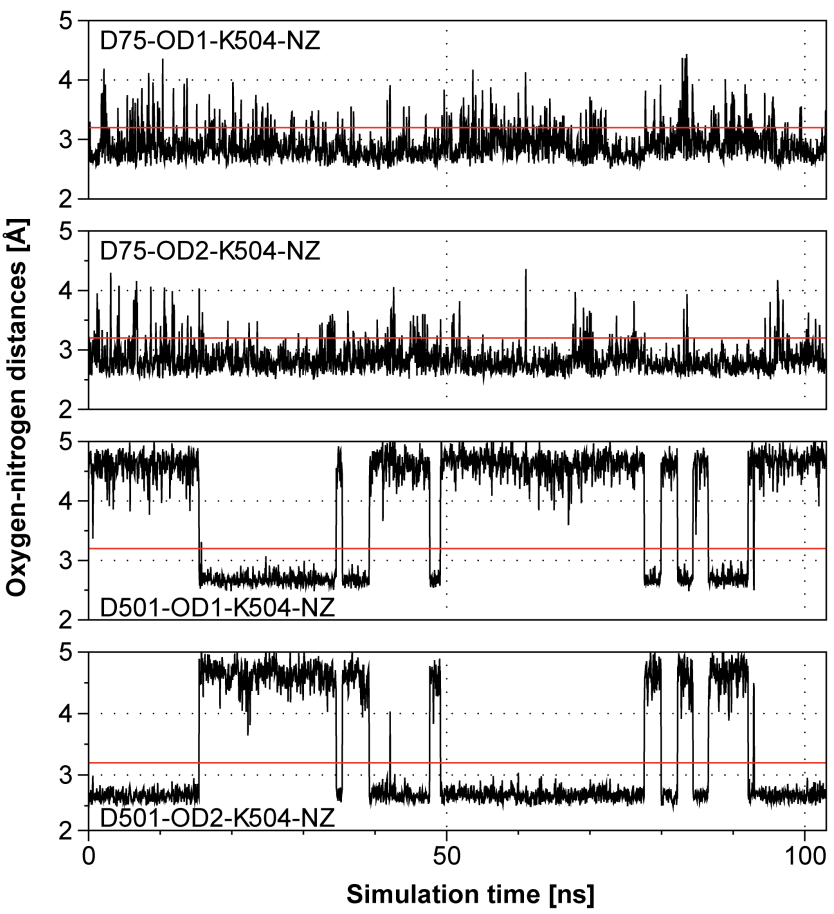
Cation binding pocket of OsHKT2;2

Figure 5: Mutation of P71 does not affect channel functionality. (A) Mean current-voltage (IV) curves and standard deviation calculated from data obtained from at least three different oocytes. (B) Representative currents measured at the indicated $\mathrm{NaCl}$ and $\mathrm{KCl}$ concentrations in Xenopus oocytes two days after cRNA injection. A pulse at holding potential (zero current level) was followed by $1 \mathrm{~s}$ voltage pulses from +20 to $-160 \mathrm{mV}$ in $-15 \mathrm{mV}$ decrements and continued with a final pulse at holding potential for $1.5 \mathrm{~s}$.

\section{OsHKT2:2-P71A}

A
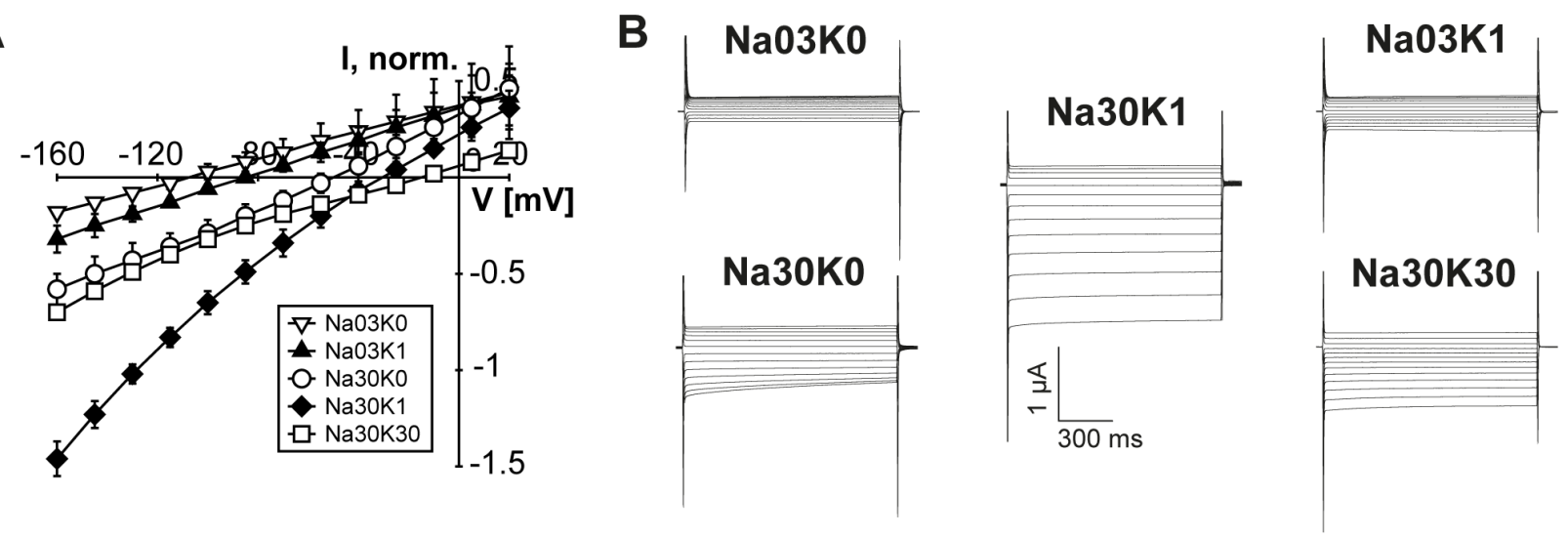
Cation binding pocket of OsHKT2;2

Figure 6: Mutations of D75 and D501 maintain channel functionality with only small changes in the kinetics. (A, C, D, F) Mean current-voltage (IV) curves and standard deviation calculated from data obtained from at least three different oocytes. (B, E, G) Currents measured at $-145 \mathrm{mV}$ at the beginning (dark grey) or end (white) of voltage pulses and of average currents over the whole voltage pulse (light grey) of D75A and D75N (B), D501A (E) and D501N (G). Currents were normalized to currents measured in Na30K1 solution. Means and standard deviations were obtained from data of three to five different oocytes.
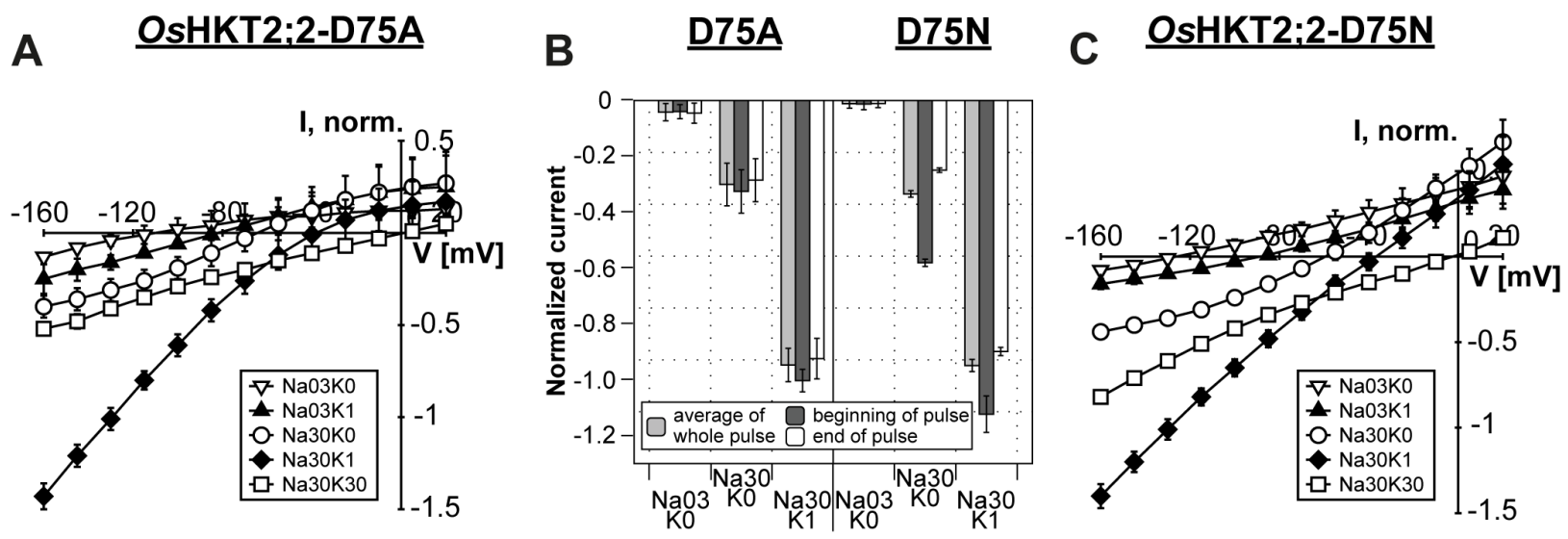

OsHKT2:2-D501A
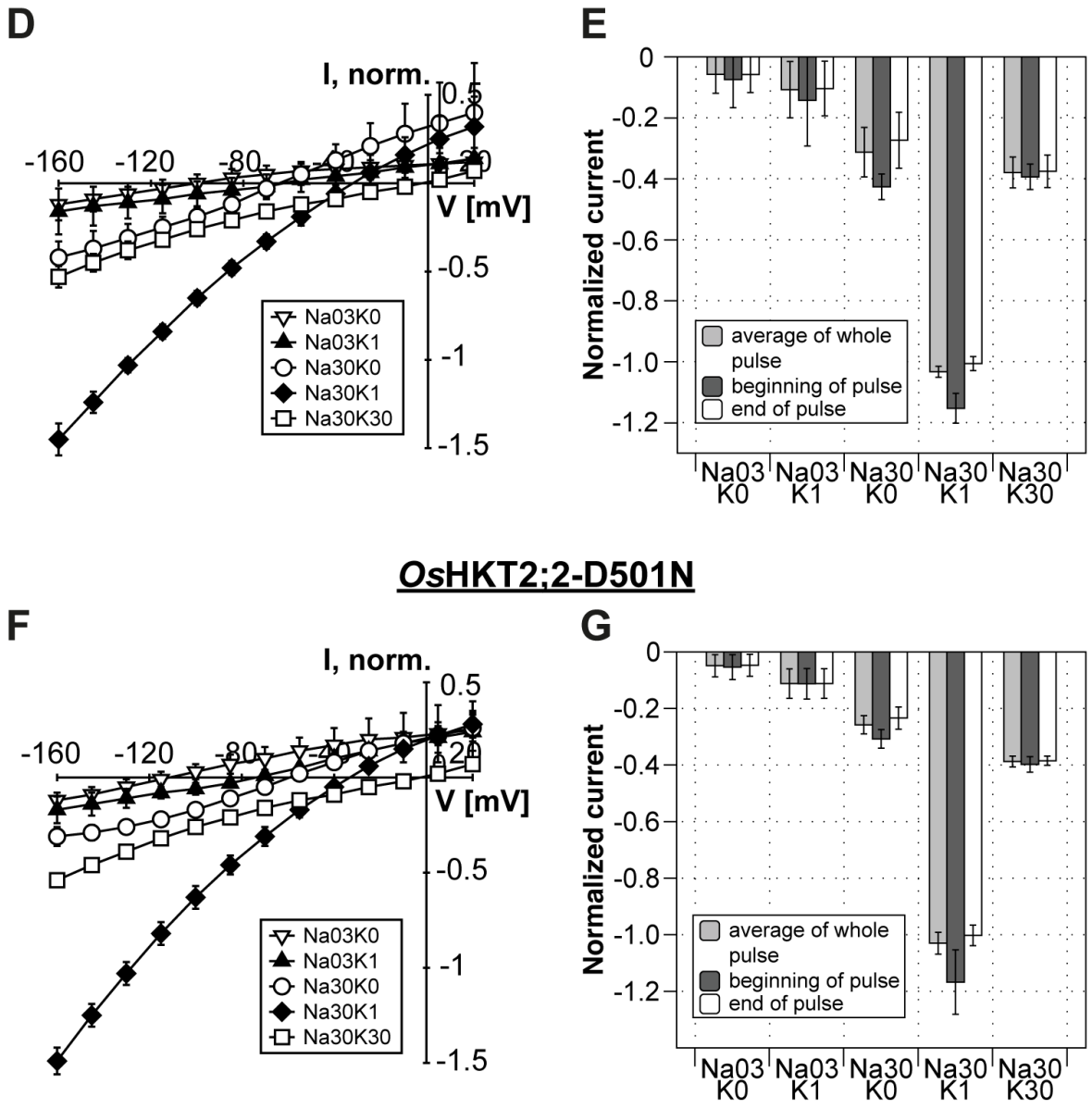
Cation binding pocket of OsHKT2;2

Figure 7: Mutations of $\mathrm{K504}$ affect conduction behaviour of $\mathrm{Na}^{+}$substantially. (A-C, E-I) Representative currents recorded in Xenopus oocytes two days after cRNA injection at indicated $\mathrm{Na}^{+}$and $\mathrm{K}^{+}$concentrations: $\mathrm{Na} 03 \mathrm{~K} 0-0.3 \mathrm{mM} \mathrm{NaCl}$ without $\mathrm{KCl}, \mathrm{Na} 30 \mathrm{~K} 0-30 \mathrm{mM} \mathrm{NaCl}$ without $\mathrm{KCl}, \mathrm{Na} 30 \mathrm{~K} 1-30 \mathrm{mM} \mathrm{NaCl}$ and $1 \mathrm{mM} \mathrm{KCl}$. A pulse at holding potential (zero current level) was followed by $1 \mathrm{~s}$ voltage pulses from +20 to $-160 \mathrm{mV}$ in $-15 \mathrm{mV}$ decrements and continued with a final pulse at holding potential for $1.5 \mathrm{~s}$. (D, J) Mean current-voltage (IV) curves and standard deviation calculated from data obtained from three to six measurements.

OsHKT2;2-K504R

A
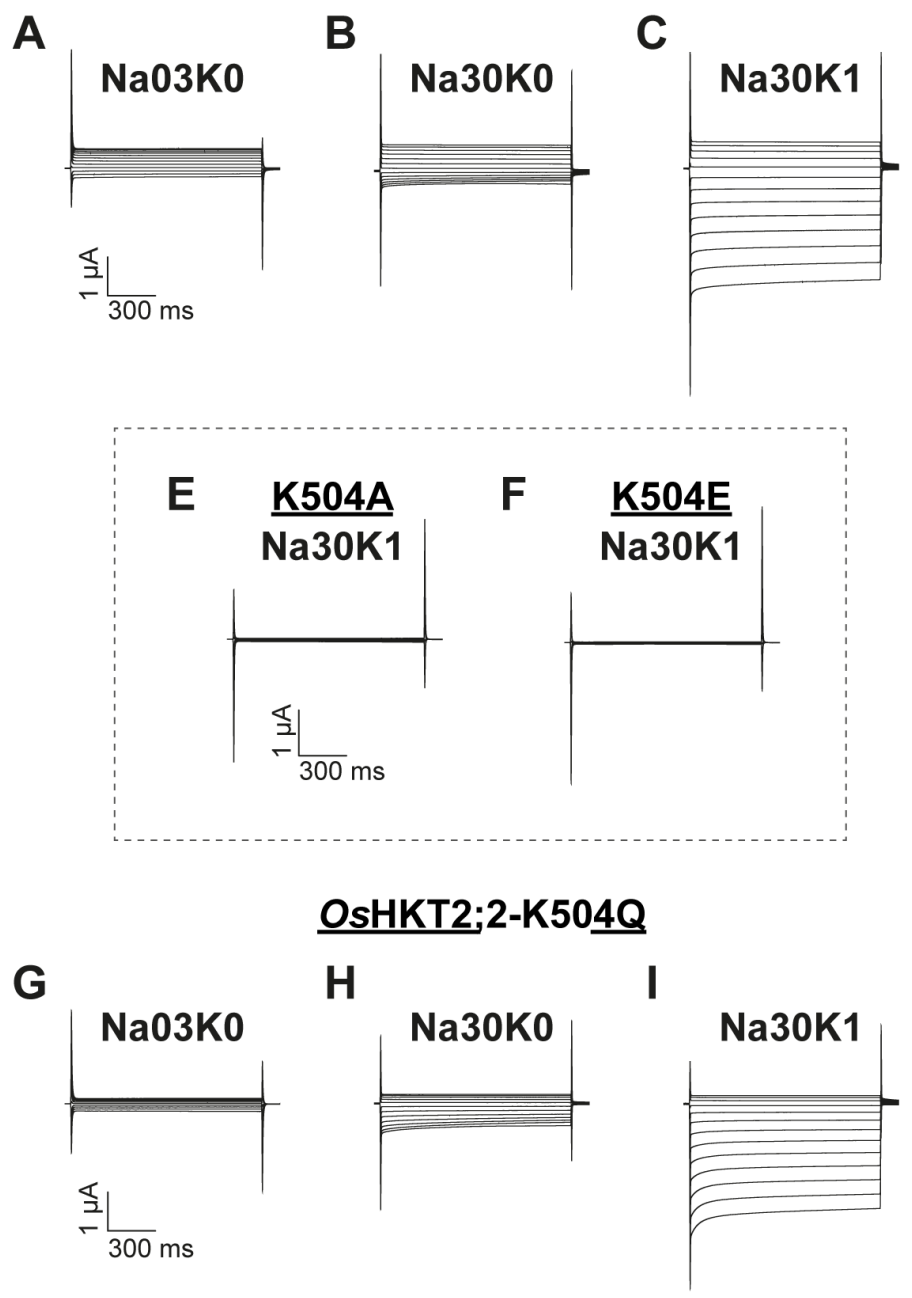
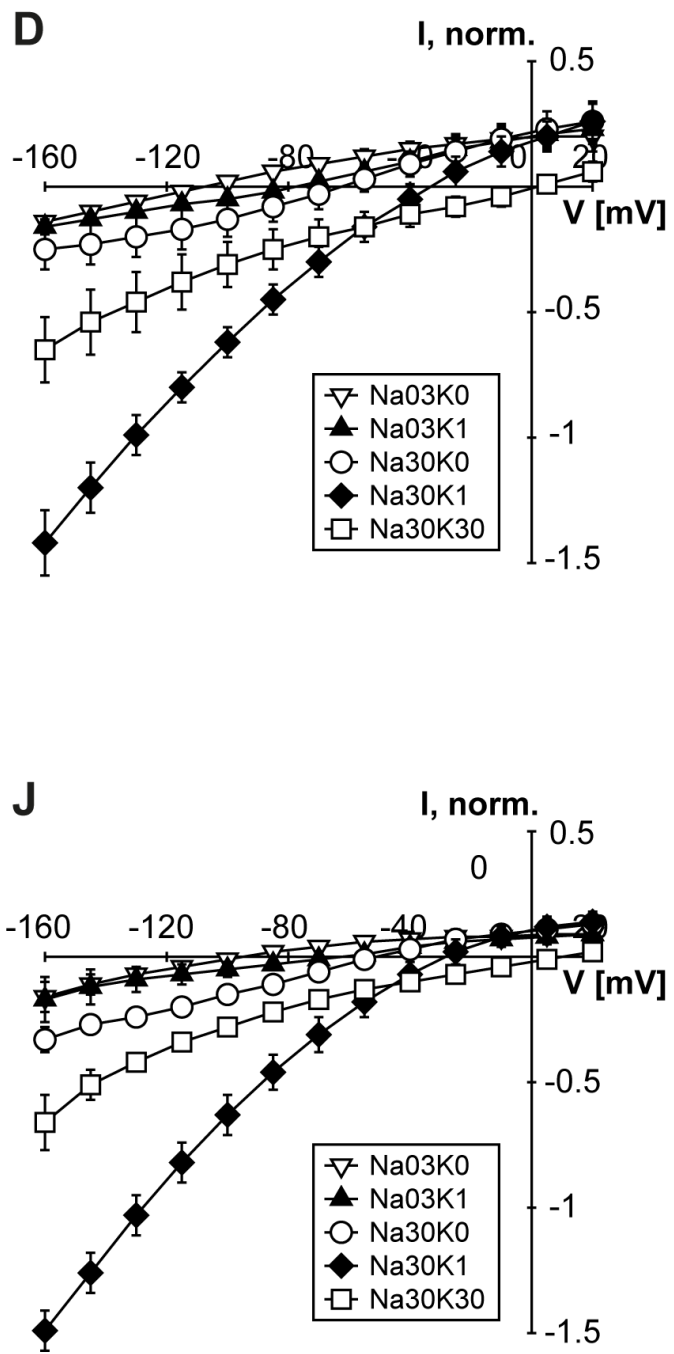
Figure 8: Binding pocket constitution of in silico mutants K504A, K504E, K504Q and K504R. (A-E) Electrostatic potential mapped to the protein surface. The cation binding pocket is highlighted and the position of P71, D75, D501 and K504 are indicated. (F-J) Representative illustration of the binding pocket constitution of all four K504 mutants and the wild type with ions approaching from the extracellular site or being inside the binding pocket.
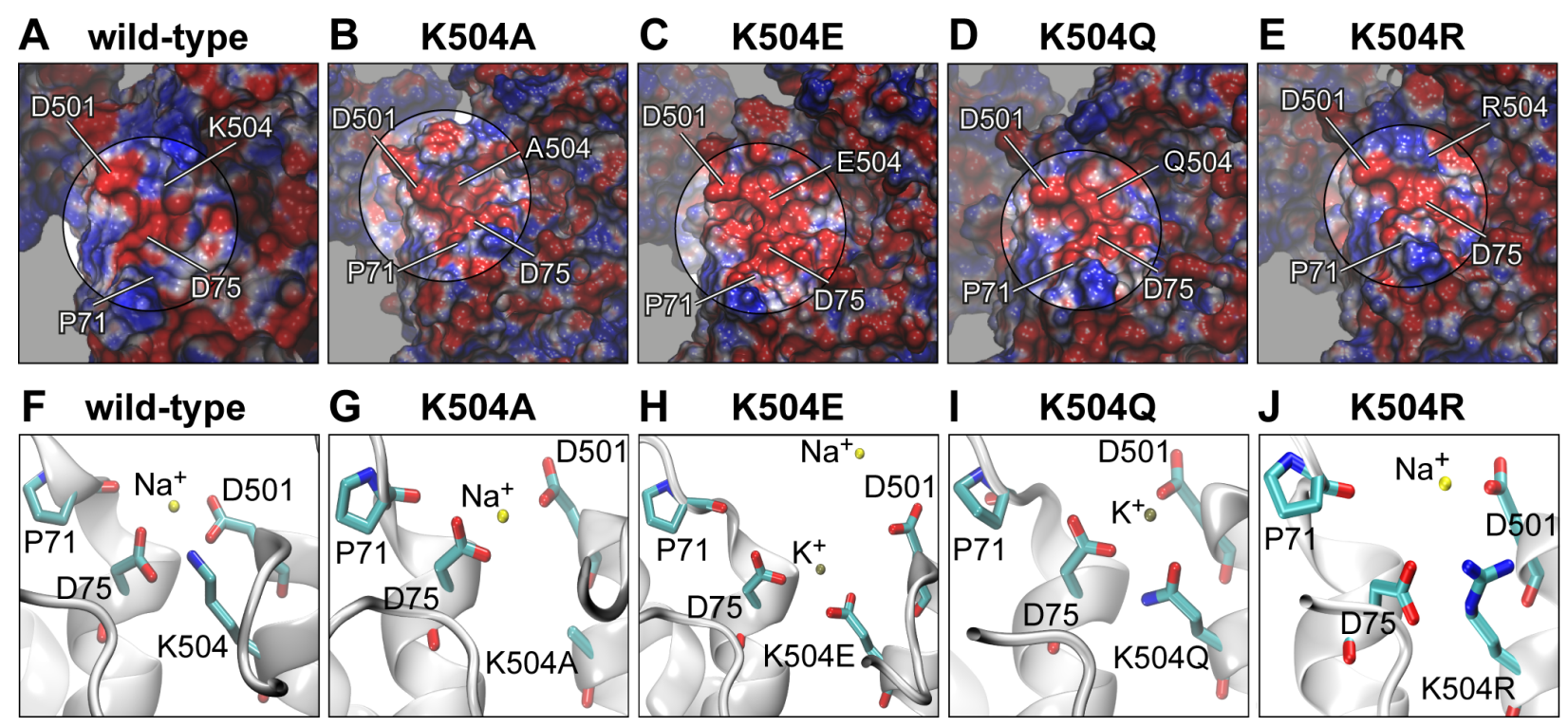